\title{
Reacciones ante las muestras de afecto entre parejas homosexuales
}

\author{
Andrea López, Ariana Sesin
}

\section{Universidad de Iberoamérica}

\section{Resumen}

Por medio de un estudio experimental de corte transversal se examina las reacciones de rechazo y homofobia hacia fotografías que dibujan muestras de afecto entre personas del mismo sexo para determinar si el nivel de religiosidad, el sexo y la edad influyen. La muestra está compuesta por 199 costarricenses, quienes completaron tres versiones de cuestionario según la pareja que se presenta. Los resultados indican que a mayor edad y mayor religiosidad se presenta un rechazo hacia las muestras de afecto y por ende homofobia. Similar, los hombres son quienes mayor discriminación presentan en comparación con las mujeres. Se comparan los resultados con investigaciones nacionales e internacional previas para ofrecer explicaciones de las asociaciones y diferencias encontradas.

Palabras clave: Homofobia, Religiosidad, Homosexualidad, Lesbianismo

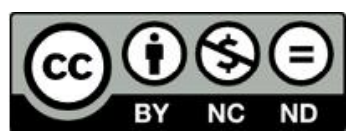




\section{Introducción}

La lucha por los derechos a la libre expresión y ejercicio de la diversidad sexual pone de manifiesto que en muchas de las sociedades en las que vivimos existe un alto grado de discriminación en torno a estas prácticas. El espacio público se ha reservado para las personas que se identifican con una sexualidad heterosexual, y se espera de las personas sexualmente diversas que no manifiesten abiertamente estas conductas. La preferencia sexual y las muestras de afecto entre personas del mismo sexo han sido causa de discriminación, debido a los valores religiosos, morales y socioculturales característicos de la sociedad costarricense.

Según estudios del CIPAC (Madrigal y Suarez, 2003; 2007) las relaciones afectivas entre personas del mismo sexo, actualmente, son vistas como algo negativo dentro de un grupo importante de la sociedad costarricense; sí como por instituciones públicas que perpetúan la discriminación, por ejemplo, grupos religiosos y leyes desfavorables que violan los derechos humanos de esta población. En Costa Rica el 90\% de la población costarricense rechaza la discriminación y dice apoyar que esta población tenga derechos. Por otro lado, en mayo del 2014 la Caja Costarricense de Seguro Social, otorgó a la población LGBTI el derecho de poder asegurarse entre ellos, situación que causó entre la población costarricense un alto nivel de conflicto y disconformidad, lo que evidencia que la realidad nacional es distinta a lo que plantea el estudio realizado por el CIPAC.

Lo que se pretende con esta investigación, es analizar la influencia del nivel de religiosidad, y variables como la edad, sexo sobre lo que piensan las personas costarricenses 
ante las muestras de afecto que se da entre parejas del mismo sexo. Lo anterior con el fin de formar una amplia visión sobre la temática, y dar pie a discusiones que ayuden a formar criterios sólidos, principalmente enfocados a contribuir con el respeto de los derechos de la comunidad LGBTI.

En Costa Rica, Arias Molina, Izquierdo Flores, Montero y Ortiz Ortiz (2008) estudiaron las muestras de afecto en una población estudiantil universitaria (14 personas), por medio de entrevistas individuales. Entre los resultados se encontró que se categorizó la homosexualidad bajo diferentes etiquetas, desde que es una preferencia o una enfermedad hasta que es una moda. Algunos participantes mostraron repulsión ante las relaciones homosexuales entre personas del mismo sexo; esto quiere decir que una mujer entrevistada sentía más repulsión por una pareja de lesbianas que hacía una de hombres y de la misma forma aplicaba para los hombres. Aparte también se vio una mayoría está en contra del matrimonio entre parejas del mismo sexo, principalmente a causa de su religión (católica) y/o valores impuestos por la familia.

Datos relacionados con lo anterior encontró Chávez Fernández (2014), entre población universitaria de 18 a 30 años de edad. Los resultados arrojan que a mayor presencia de homofobia hay mayor nivel de religiosidad, asimismo, la presencia de contacto con personas homosexuales o lesbianas baja la probabilidad de que se presente la discriminación. Resultados similares se encuentran en Rodríguez (2012), añadiendo que el género masculino presenta mayor homofobia. 
En el escenario internacional, España Albelda, Guerrero Roca, Farre, Canella Soler y Abós (2001); por medio de una escala para detectar la homofobia se encuesta a 80 personas universitarias en España. Dentro de los resultados se resalta que 64\% de la población estuvo en desacuerdo o se sentiría incómodo en presencia de un homosexual, mientras que un $63 \%$ estuvo de acuerdo en que se incluya a la homosexualidad en la educación sexual. Tal como se ha confirmado con otros estudios las mujeres presentan manos homofobia; sucede igual en el caso de personas que tienen posturas políticas más abiertas.

También Fernández Rodríguez y Calderón Squiabro (2014) exploraron las actitudes de rechazo y distancia social hacia las personas gays y lesbianas en 565 universitarios. Se utilizó una escala para medir Prejuicio y otra escala para medir Distancia Social. Los participantes reflejaron niveles moderados de prejuicio y distancia social hacia las personas gays y lesbianas. Los hombres significativamente más prejuicio y distancia social que las mujeres. Un resultado interesante es que las personas que asisten con regularidad a la iglesia mostraron más prejuicio y distancia social que los que no asisten.

Siguiendo la línea de investigación, Campos Cantore, Rodríguez y Trías (2008) realizaron un estudio en Venezuela en el cual se encontró de manera principal que el estudiantado de la universidad involucrada presenta una actitud neutra hacia la homosexualidad. Bajo la variable de género se encontró nuevamente que las mujeres presentan una mayor aceptación o tolerancia hacías estas personas. Un dato interesante y nuevo fue que personas en carreras como Computación y Bioanálisis presentaron una actitud menos favorable que de otras carreras en el área de las humanidades.

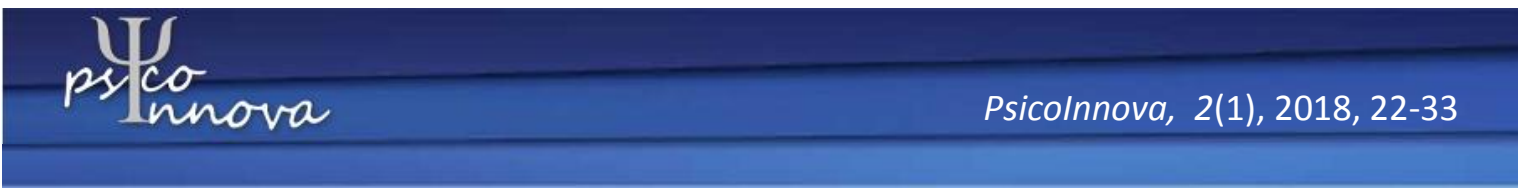


En Europa (43) países fueron estudiados en torno a las creencias de homonegatividad y el sentido de pertenencia a una religión. Se obtiene de la aplicación de cuestionarios que el $50 \%$ de la muestra consultada presenta rechazo hacia la población homosexual y que la afiliación religiosa está asociada positivamente a la homonegatividad (Dobler, 2015).

Se aprecia que en los estudios consultados las variables utilizadas son múltiples, pero abarcan el tema de la discriminación hacia personas homosexuales y lesbianas. Dentro de la discriminación existen varias formas, el rechazo tácito hacia la idea de que existan personas que tienen esta elección, el rechazo a las muestras de afecto entre ellos o bien a la expresión de la sexualidad ante otros. Aunado a lo anterior, se liga la afiliación religiosa con la presencia de actitudes y creencias discriminatorias. Cabe resaltar que las variables demográficas como el sexo, son de peso en todos los estudios realizados. Estas investigaciones son fuente de información valiosa para comprender el fenómeno en estudio y arrojar explicaciones posibles para la presente investigación.

\section{Método}

El estudio que se expone es de tipo experimental, ya que se evaluá la actitud hacia las muestras de afecto entre parejas del mismo sexo por medio de la aplicación de un cuestionario que muestra el comportamiento en cuestión. El diseño experimental incluye tres cuestionarios, uno en el que se presenta a dos hombres, otro a dos mujeres y el tercero a una pareja heterosexual. Los datos se recogieron en línea, cuyo único criterio de inclusión era que fueran costarricenses. La muestra estuvo formada por 199 personas, de las cuales el 
$68.8 \%$ fueron mujeres y $31.2 \%$ de los casos fueron hombres, la media de edad es de 27.81 $(\mathrm{DS}=11.19)$.

El análisis de datos se realiza por medio del paquete estadístico para las ciencias sociales (SPSS v.23). Los análisis incluyen análisis descriptivos, anovas, pruebas de diferencias de medias y correlaciones. El nivel de significancia se ubica en 0.05.

\section{Resultados}

Con respecto a la religión, $62 \%$ son cristianos católicos, $45 \%$ cristianos protestantes y $14 \%$ ateos o agnósticos. En el caso de la orientación sexual de los participantes el 86.4\% se identifica con la orientación heterosexual, y el $12 \%$ indica identificarse con la homosexualidad, bisexualidad y lesbianismo.

Se comparó el nivel de religiosidad, homofobia y la edad de los participantes, los resultados obtenidos indican que, a mayor edad, mayor grado de homofobia $(r=.222, p=.000)$; y a mayor nivel de religiosidad, mayor grado de homofobia $(r=.390, p=.000)$.

Se realiza un anova de medidas repetidas para determinar las diferencias según la orientación sexual en el nivel de rechazo a las muestras de afecto entre parejas del mismo sexo. En la tabla 1 se muestra las medias y desviaciones estándar para cada grupo. 
Tabla 1. Medias y Desviaciones Estándar para los niveles de desagrado hacia las muestras de afecto entre personas del mismo sexo

\begin{tabular}{llll}
\hline Desagrado & Parejas & Parejas Homosexuales & Parejas \\
& Heterosexuales & Lesbianas \\
\hline Mujer & & & \\
M & .34 & 2.74 & 2.63 \\
SD & .87 & 3.30 & 3.06 \\
Hombre & & & \\
M & .65 & 2.82 & 1.86 \\
SD & 2.24 & 3.17 & 3.02 \\
\hline
\end{tabular}

El análisis muestra un efecto significativo en la orientación sexual de la pareja mostrada $\left(\mathrm{F}_{2,193}=10.501, \mathrm{p}=.000\right)$. Las pruebas post hoc utilizando la corrección Bonferroni muestran que el observar muestras de afecto de parejas heterosexuales $(\mathrm{M}=.496,95 \% \mathrm{CI}[-$ $2.41,1.234])$ genera menor desagrado que el observar parejas homosexuales $(\mathrm{M}=2.780,95 \%$ CI $[2.080,3.480])$ y lesbianas $(\mathrm{M}=2.246,95 \%$ CI $[1.478,3.015])$.

Un análisis de efectos simple muestra diferencias estadísticamente significativas en los niveles de desagrado de las mujeres al observar muestras de afecto de una pareja homosexual $(\mathrm{p}=.000)$ o lesbiana $(\mathrm{p}=.001)$ en comparación con las parejas heterosexuales. En el caso de los hombres se encuentran mayores niveles de desagrado al observar muestras de afecto de parejas homosexuales ( $\mathrm{p}=.013)$ en comparación con las parejas heterosexuales. 


\section{Discusión}

Primeramente, se resalta que la hipótesis planteada para este estudio, en donde se proponía que los participantes iban a mostrar rechazo hacia las muestras de afecto entre personas del mismo sexo se verifica. En el caso de los hombres, al igual que en todos los estudios consultados como antecedentes, se descubre que experimentan un mayor rechazo hacia las muestras de afecto entre personas del mismo sexo, en este caso hombres. Al igual, se confirma que en Costa Rica, las mujeres presentan mayor tolerancia hacia estas muestras; sin embargo, esto no significa que acepten las muestras de afecto entre homosexuales completamente (Campo Cantore, Rodríguez y Trías, 2008; Arias-Molina, Izquierdo-Flores).

Si bien es cierto no se encontraron antecedentes que reflejen diferencias de edad, la presente investigación incluyó esta variable y se corroboró la hipótesis de que a mayor edad mayor será el nivel de homofobia. Lo anterior puede deberse a varias razones, las generaciones pasadas tuvieron un contacto y una educación con respecto al tema limitada y moldeada hacia el rechazo. En años pasados se creía que la homosexualidad era una enfermedad, que las personas homosexuales eran personas malas y además si se combina la edad con la religiosidad, es claro que la iglesia ha perpetuado visiones ortodoxas hacia la relación sexual, limitándola a las personas heterosexuales en el marco del matrimonio. Este estudio presenta una media de edad bastante joven, sin embargo, si se encuestó a personas de mayor edad que pudieron tener un peso sobre la relación. En el caso de población joven, a pesar de que pueden mantener sus creencias religiosas, la visión de derechos humanos se ha generalizado e instruido desde pequeños en escuelas y colegios. Esto podría estar

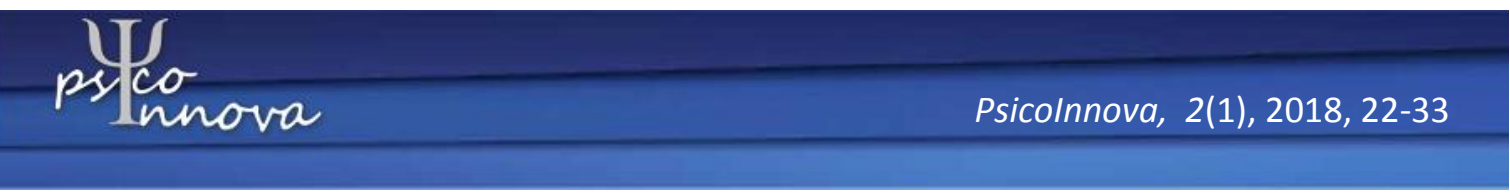


beneficiando las actitudes de aceptación y tolerancia entre los jóvenes hacia la población LGBTI (PEW Research Center Religion and Public Life, 2014).

Cabe destacar que son las personas que se identifican con la religión cristiana protestante las que presentan mayor nivel de homofobia ante la presentación de las imágenes, este resultado se ha obtenido de otras investigaciones anteriormente mencionadas (Cháves Fernández, 2014); España Albeda, Farre, Canela Soler y Abós, 2001), Dobler, 2015; PEW Research Center Religion and Public Life, 2014). Los autores del Pew Research Center proponen que a raíz de la apertura y actitud de respeto y acogida en la iglesia que ha mostrado el papa Francisco, la comunidad católica latina ha optado por seguir sus recomendaciones y de esta forma ha bajado los niveles de discriminación; esta es una hipótesis que debe ser confirmada, comparando con otras poblaciones católicas de países no latinoamericanos.

Por último, las investigaciones consultadas y la presente demuestran que a pesar que los esfuerzos por incorporar el respeto a los derechos humanos y brindar información a las población todavía las parejas del mismo sexo sufren mucha discriminación ya sea que su sexualidad sea abierta o en privado. Lo cual genera en la población LGBTI un fuerte impacto y daño a nivel emocional y psicológico, a tal punto que puede presentarse casos de suicidio. En una investigación de Madrigal y Suárez sobre suicidio en la población homosexual costarricense (2002), se logra ver cómo altos porcentajes de la población LGBTI (22\%) ha terminado en acabar con su vida y un $42 \%$ lo ha intentado. Lo más alarmante es que la mayoría de estas personas no recibieron o reciben atención o apoyo psicológico a tiempo o posterior al intento. Todas las personas tienen derecho a vivir su vida en armonía con los 
derechos humanos y como sociedad, sin importar la orientación sexual de las personas, es nuestro deber mostrar respeto, ya que del respeto se derivan muchas otras cosas que van abriendo poco a poco la brecha de la tolerancia y la inclusión de esta población en la sociedad.

\section{Referencias}

Arias-Molina, A. Izquierdo-Flores, C. Montero, N \& Ortiz-Ortiz, A. (2008). Percepciones de la población universitaria de la Universidad de Costa Rica, sede Rodrigo Facio, hacia las relaciones afectivas entre personas del mismo sexo. Recuperado de: http://cqinvestigo.wdfiles.com/local--

files/cualitativas/Percepciones\%20de\%20la\%20poblaci\%C3\%B3n\%20universitaria $\% 20 \mathrm{de} \% 201 \mathrm{a} \% 20$ Universidad $\% 20 \mathrm{de} \% 20$ Costa $\% 20$ Rica, $\% 20$ sede $\% 20$ Rodrigo $\% 20$ Facio,\%20hacia\%20las\%20relaciones $\% 20$ afectivas $\% 20$ entre $\% 20$ personas $\% 20 \mathrm{del} \%$ 20mismo\%20sexo\%20.pdf

Madrigal, F. y Suárez. (2007). Percepción de estudiantes universitarios sobre la homo/lesbofobia en Costa Rica. Para Diagnóstico, con enfoque de género, sobre la situación del estigma, la discriminación y la homofobia en Costa Rica. CICAP: San José Costa Rica. Recuperado de: http://www.cipacdh.org/pdf/diagnostico_costa_rica.pdf 
Madrigal, F. y Suarez, D. (2003). Diagnóstico Situacional de la poblaciónGay/lésbica/bisexual y transgénero de Costa Rica. Recuperado de: http://lib.ohchr.org/HRBodies/UPR/Documents/Session6/CR/CIPAC-

DDHH_CRI_UPR_S06_2009_S.pdf

Cháves-Fernández, W. (2014). Grado de Homofobia y Lesbofobia en los y las estudiantes entre 18 y 30 años de la Universidad Nacional. (Tesis para obtener el grado de licenciatura en psicología). Universidad Nacional, Costa Rica.

España-Albelda, E., Guerrero-Roca, A., Farré, J. M., Canella-Soler, J. \& Abós, R. (2001). La homofobia en el medio universitario. Un estudio empírico. Medicina. Psicosomática y medicina de enlace, 57, 41-55.

Fernández-Rodríguez, M., y Calderón-Squiabro, J. (2014). Prejuicio y distancia social hacia personas homosexuales por parte de jóvenes universitarios. Revista Puertorriqueña De Psicología, 25(1), 52-60. Recuperado de http://reps.asppr.net/ojs/index.php/reps/article/view/230

Campo-Cantore, K. Rodríguez, M.G. y Trías, L. (2008). Actitudes de los estudiantes universitarios hacia la homosexualidad. Rev. Esc. Psicol. Univ. Cent. Venez, 27(2), 91-120. 
Doebler, S. (2015). Relationships between Religion and two forms of Homonegativity in Europe, A Multilevel Analysis of Effects of Believing, Belonging and Religious Practice. Plos One, 10(8). doi: 10.1371/journal.pone.0133538

Rodríguez, M. (2012). Factores psicosociales predictores de la homofobia en estudiantes universitarios. (Tesis para obtener el grado de licenciatura en psicología).

Pew Research Center (2014). Religion in Latin America: Widespread Change in Historically Catholic Region. Recuperado de: http://www.pewforum.org/2014/11/13/religion-inlatin-america/

Madrigal, F. y Suárez, D. (2002). Diagnóstico Situacional de la poblaciónGay/lésbica/bisexual y transgénero de Costa Rica. Recuperado de: http://lib.ohchr.org/HRBodies/UPR/Documents/Session6/CR/CIPAC-

DDHH_CRI_UPR_S06_2009_S.pdf

Madrigal, F. y Suarez. (2000). Suicidio en la población homosexual costarricense. Recuperado de: http://www.cipacdh.org/cipac_articulo_completo.php?art=66 